\title{
DESENVOLVIMENTO DE UMA TECNOLOGIA PARA A DETECÇÃO E INTERRUPÇÃO DO EPISÓDIO DA APNÉIA DA PREMATURIDADE
}

\author{
Vania Carla Camargo ${ }^{1}$, Sandra Honorato da Silva², Percy Nohama³, Mardson de Freitas Amorim ${ }^{4}$
}

\footnotetext{
${ }^{1}$ Mestre em Tecnologia em Saúde. Docente do Curso de Enfermagem da Faculdade Unibrasil. Paraná, Brasil. E-mail: vaniacamargo10@gmail.com

${ }^{2}$ Doutora em Enfermagem. Professor Titular do Curso de Enfermagem e do Mestrado em Tecnologia em Saúde da Pontifícia Universidade Católica do Paraná (PUC-PR). Paraná, Brasil. E-mail: sandra.honorato@pucpr.br

${ }^{3}$ Doutor em Engenharia Elétrica. Professor Titular do Departamento de Engenharia de Computação e do Programa de PósGraduação em Tecnologia em Saúde da PUC-PR. Professor do Departamento de Eletrônica e do Programa de Pós-Graduação em Engenharia Elétrica e Informática Industrial da Universidade Tecnológica Federal do Paraná. Paraná, Brasil. E-mail: percy@ppgts.pucpr.br

${ }^{4}$ Doutor em Engenharia Biomédica. Professor Adjunto da Universidade Federal do Ceará. Ceará, Brasil. E-mail: mardson@ ufc.br
}

RESUMO: Trata-se de um estudo de desenvolvimento e avaliação de um sistema de detecção e interrupção do episódio de apnéia da prematuridade no recém-nascido pré-termo, utilizando o estimulo vibrotátil para reversão. O sistema foi testado em quatro recém-nascidos pré-termos, em 18 episódios apnéicos observados diretamente nas unidades de terapia intensiva. Para interromper a apnéia o mesmo recém-nascido foi submetido, distintamente, ao estímulo manual e vibratório em $250 \mathrm{~Hz}$ por quatro segundos. Os dados foram tratados por estatística descritiva. O método de detecção da apnéia mostrou-se satisfatório e o método de interrupção da apnéia por estímulo vibratório promoveu o retorno dos movimentos respiratórios, em nove das 10 apnéias. A tecnologia desenvolvida e testada embora demande continuidade de estudos, vislumbra uma tecnologia promissora para a melhoria da qualidade assistencial em neonatologia.

DESCRITORES: Apnéia. Prematuro. Tecnologia em saúde.

\section{DEVELOPMENT OF A TECHNOLOGY FOR THE DETECTION AND INTERRUPTION OF PREMATURE APNEA EPISODES}

\begin{abstract}
This is a study of the development and evaluation of a system to detect and interrupt premature apnea episodes in newborn pre-terms, using vibrotactile stimulus as reversal. The system was tested in four preterm newborns involving 18 directly observed apnea episodes in the intensive care units. In order to stop apnea, the newborn was submitted, separately, to manual stimulus and guide vibration at $250 \mathrm{~Hz}$ by four segundos. The data was analyzed with descriptive statistics. The method of apnea detection was shown to be satisfactory and the method of interrupting sleep apnea through vibratory stimulation promoted the return of respiratory movements in nine of 10 apneas. The technology developed and tested, while it demands continuity of studies, gleams a promising technology for improving the quality care in neonatology.
\end{abstract}

DESCRIPTORS: Apnea. Premature. Technology in health.

\section{DESARROLLO DE UNA TECNOLOGÍA PARA LA DETECCIÓN E INTERRUPCIÓN DE LA APNEA DEL PREMATURO}

\begin{abstract}
RESUMEN: Es un estudio sobre el desarrollo y la evaluación de un sistema de detección e interrupción del episodio de apnea del prematuro en los recién nacidos a término, utilizando el estímulo vibrotáctil de revertir. El sistema fue probado en cuatro recién nacidos prematuros, en 18 episodios de apnea observados directamente en las unidades de cuidados intensivos. Para detener la apnea del recién nacido, el mismo fue sometido, por separado, a la estimulación manual y a las vibraciones a $250 \mathrm{~Hz}$ por cuatro segundos. Los datos fueron procesados por la estadística descriptiva. El método de detección de la apnea ha demostrado ser satisfactorio y el método de interrupción de la apnea por la estimulación vibratoria promovió el retorno de los movimientos respiratorios, en nueve de las 10 apneas. La tecnología desarrollada y probada aunque requiere la continuidad de estudios, se ve como una tecnología prometedora para mejorar la calidad de la atención en neonatología.
\end{abstract}

DESCRIPTORES: Apnea. Prematuro. La tecnología en salud. 


\section{INTRODUÇÃO}

Nascem no mundo, por ano, cerca de 20 milhões de pré-termos e destes, um terço morre antes de completar um ano de vida. Acrescenta-se a este dado a estimativa que $50 \%$ dos pré-termos com menos de $1.500 \mathrm{~g}$, internados em Unidade de Terapia Intensiva Neonatal, (UTIN) desenvolvem Apnéia da Prematuridade (AP). ${ }^{1}$ Considera-se pré-termo o bebê nascido abaixo de 36 semanas e 6 dias gestacionais. ${ }^{2}$

Os Recém-nascidos Pré-termos (RNPTs) são os mais propensos a desenvolver apnéia e os episódios aumentam com o decréscimo da idade gestacional. Aproximadamente $25 \%$ de todos os RNPTs com menos de $1.800 \mathrm{~g}$, ao redor de 34 semanas gestacionais apresentarão pelo menos um episódio apnéico, enquanto que a maioria dos muito pequenos, menores de 30 semanas gestacionais sofrerão ataques apnéicos ocasionais. ${ }^{2}$

A apnéia é definida como sendo uma pausa da respiração por 20 segundos ou mais, ou uma pausa respiratória inferior a 20 segundos, acompanhada de bradicardia com diminuição da frequência cardíaca em $20 \%$ e queda de saturação de oxigênio, menor que $85 \%$, por mais de cinco segundos. ${ }^{3}$

As grandes descobertas científicas dos últimos 30 anos geraram poucas mudanças nas estratégias de manejo da apnéia e como consequência uma escassez de novas tecnologias no cuidado aos recém-nascidos pré-termos que apresentam este quadro. ${ }^{4-5}$

Um recurso existente para o manejo da apnéia é a estimulação manual no corpo do bebê, realizada pela equipe multiprofissional de saúde. A implementação dessa manobra demanda recursos humanos em número suficiente na unidade, para que imediatamente à ocorrência da apnéia, seja realizada a interrupção do episódio. ${ }^{4}$

Como fatores negativos da interrupção da apnéia por estimulação manual citam-se o risco da contaminação cruzada ${ }^{5}$ e a produção de níveis iguais ou até mais elevados de estresse e sensação dolorosa, considerando que o simples estímulo manual pode produzir dor no bebê com a excessiva manipulação destes Recém-nascidos (RNs) nas UTINs. ${ }^{6-7}$ Acrescenta-se que esta estimulação depende da ação humana direta, fadada, algumas vezes, à falhas, negligência, imperícia, e agravada pelo número insuficiente de profissionais para o atendimento RNs nas UTINs. Esses fatores podem retardar uma ação eficaz de estímulo ao bebê apnéico, acarretando-lhe danos.
No contexto das estratégias de manejo das apnéias, a utilização de fármacos produz efeitos colaterais conhecidos, decorrentes do uso da teofilina como taquicardia, sangramento digestivo alto, aumento da diurese, hiperglicemia e hipercalciúria. ${ }^{8}$ São conhecidos também, os efeitos negativos do uso da pressão positiva em vias aéreas e da ventilação mecânica. Os inconvenientes destes métodos decorrem do risco de lesão local que as cânulas podem produzir, provocando necrose tecidual, estenose nasal e deformidades. ${ }^{9}$

Considerando as iatrogenias que o atual manejo da apnéia do RN pode causar e que a produção de tecnologia deve ser um empreendimento alicerçado sobre uma necessidade, como um problema a ser resolvido, ${ }^{10} \mathrm{o}$ desenvolvimento de estudos que busquem novas tecnologias para o manejo da apnéia torna-se imperioso.

Este estudo tem como objetivo desenvolver e avaliar uma tecnologia de detecção e interrupção do episódio de apnéia no RN, baseada nos parâmetros de frequência de pulso e Saturação de Oxigênio $\left(\mathrm{SpO}_{2}\right)$, simultaneamente, e utilizando o estímulo vibrotátil para a reversão de tal episódio.

Esta tecnologia de detecção e interrupção do episódio apnéico prevê a estimulação do bebê, somente quando estritamente necessário, minimizando assim, os efeitos provocados por estímulos e manuseios desnecessários.

\section{MÉTODO}

Trata-se de um estudo de desenvolvimento e avaliação de um sistema para detecção e interrupção de episódio apnéico que contemplou duas etapas, sendo a primeira direcionada a concepção e desenvolvimento do sistema e a segunda a avaliação com os RNs.

A etapa de avaliação do sistema de detecção e interrupção da apnéia envolveu quatro RNPTs, diagnosticados com AP, internados em UTINs, de Curitiba-PR. Para a seleção dos bebês foram adotados os seguintes critérios: ambos os sexos, nascidos em idade gestacional abaixo de 36 semanas e seis dias gestacionais, peso inferior ou igual a $2500 \mathrm{~g}$, sem anomalias congênitas, desordens cardíacas, ou outra patologia associada, sem terem sido expostos, intra-útero a drogas ilícitas ou álcool.

Foi estabelecido um protocolo para o desenvolvimento da avaliação. O equipamento era testado antes do início do experimento e logo após o término do mesmo, para comprovação 
do funcionamento do mesmo. O RN tinha os parâmetros de Frequência de Pulso (FP) e $\mathrm{SpO}_{2}$ mensurados pelo oxímetro de pulso do sistema Anjo, ficando desligado o oxímetro da UTIN. O sensor do oxímetro era fixado no pé do bebê, na parte anterior da região plantar do pé. O local de fixação do sensor era alternado a cada quatro horas, de acordo com a recomendação do fabricante. Para a fixação do sensor, foram utilizadas gaze e fita adesiva, não sendo colado nada diretamente sobre a pele do bebê.

Depois de monitorado o $\mathrm{RN}$, o vibrador era colocado na região ântero-superior do tórax, sendo fixado por meio de uma malha ortopédica. Não foi utilizado nenhum tipo de adesivo na sua pele. Nenhum bebê apresentou qualquer alteração na pele, no local onde foi fixado o vibrador.

O mesmo bebê foi submetido aos dois métodos de reversão de apnéia, o estímulo vibrotátil e o estímulo manual em momentos distintos. Foi determinado que na primeira apnéia observada pela pesquisadora, o método utilizado seria o vibratório e na segunda, o estímulo manual e assim, alternadamente, nos próximos episódios que o bebê apresentasse.

O estímulo vibratório foi programado para ter seu acionamento automático dado pelos valores pré-determinados de $\mathrm{FPeSpO}_{2^{\prime}}$, sem a interferência da pesquisadora ou da equipe. Na ocorrência da apnéia, a pesquisadora ocupava-se somente em realizar a observação e registrar os dados do evento em impresso elaborado para o estudo. A responsabilidade da intervenção direta do estímulo manual era da equipe multiprofissional da UTIN.

Para o estímulo vibratório foi estabelecido um tempo de vibração de 4 segundos, com uma frequência de $250 \mathrm{~Hz}$. Após o estímulo era esperado por até 10 segundos o retorno dos movimentos respiratórios.

O profissional de enfermagem responsável pelos cuidados integrais do bebê estava orientado para que, na ocorrência de uma apnéia, o estímulo manual fosse aplicado da seguinte maneira: posicionar seus dedos polegar e indicador sobre o apêndice xifóide do bebê e deslizá-los delicadamente pelos bordos inferiores dos últimos arcos costais, até as laterais do tórax e retornando com os mesmos movimentos até novamente chegar ao apêndice xifóide. Estes movimentos deveriam ser realizados até o profissional perceber o retorno dos movimentos respiratórios.

Foram estabelecidas como variáveis a serem observadas em cada avaliação realizada no RN: tipo de estímulo; intervalo de tempo entre o início da apnéia e o início do estímulo (em segundos); duração do estímulo (em segundos); intervalo de tempo para o retorno dos movimentos respiratórios após o término do estímulo (em segundos); intervalo de tempo para início do aumento da FP após o retorno dos movimentos respiratórios (em segundos); intervalo de tempo entre o retorno dos movimentos respiratórios e o início do aumento da $\mathrm{SpO}_{2}$ (em segundos); intervalo de tempo para atingir $81 \%$ de $\mathrm{SpO}_{2}$ após o retorno dos movimentos respiratórios (em segundos); intervalo de tempo para atingir $88 \%$ de $\mathrm{SpO}_{2}$ após o retorno dos movimentos respiratórios (em segundos); intervalo de tempo para atingir o pico de FP após o início dos movimentos respiratórios em segundos e valor da frequência de pulso durante seu pico (batimentos por minuto).

Neste estudo, a observação do tempo para se atingir $81 \%$ de $\mathrm{SpO}_{2}$ considerou $80 \%$ como valor diagnosticador de um episódio apnéico. Assim, a obtenção da $\mathrm{SpO}_{2}$ em $81 \%$, foi considerada como o marco do estado de não-apnéia do RN.

Foram registrados todos os valores de FP inferiores a $120 \mathrm{bpm}$ e a $\mathrm{SpO}_{2}$ inferiores a 92\%; hora, minuto e segundo do estímulo vibratório; hora, minuto e segundo da duração do estímulo manual; hora, minuto e segundo da ocorrência da apnéia no momento do estímulo manual, o intervalo de tempo entre a detecção da apnéia e o início da estimulação manual pela equipe, bem como, intervalo de tempo para o restabelecimento de cada parâmetro à normalidade.

O critério de eficácia para os dois métodos utilizados foi considerado como sendo o momento em que a respiração voltasse a ser espontânea e regular, os parâmetros de $\mathrm{SpO}_{2}$ atingissem um valor igual ou maior que $88 \%$, que a frequência cardíaca atingisse $100 \mathrm{bpm}$ ou mais, sendo considerados esses critérios para todos os RNs, independentemente de sua idade gestacional ao nascimento. Esses parâmetros foram adotados com base em autores consultados. ${ }^{2}$ A observação direta foi a técnica utilizada para se constatar o retorno dos movimentos respiratórios, sendo à seguir registrada.

Devido à falta de padronização do procedimento de estímulo manual nas UTINs adotou-se o procedimento utilizado em uma das unidades onde a pesquisa foi realizada. Não foi encontrado um protocolo escrito de estimulação manual nas UTINs envolvidas no estudo.

O alarme sonoro soava quando um dos parâmetros, $\mathrm{FP}$ ou $\mathrm{SpO}_{2}$, apresentasse alteração. Ao soar 
o alarme, a equipe se mobilizava para atendimento ao bebê e chegando à beira da incubadora, complementava a observação da ocorrência ou não da apnéia, antes de iniciar as medidas de reversão.

Quando da ocorrência do estímulo vibratório, o bebê permanecia monitorado pelo oxímetro e os alarmes continuavam a soar normalmente, sendo o alarme da frequência de pulso independente do alarme de $\mathrm{SpO}_{2}$. Após o estímulo vibratório ter sido dado, o bebê era observado pela equipe da UTIN que aguardava, por até 10s, o retorno dos movimentos respiratórios, antes de acionar alguma outra medida para o retorno da respiração.

Na condição dos movimentos respiratórios não retornarem em até 10s após a aplicação do estímulo vibrotátil, ficava a critério dos profissionais da UTIN a escolha da medida a ser acionada, bem como a intervenção direta para o restabelecimento do quadro. O resultado do experimento vibrotátil, neste caso, seria considerado sem sucesso.

Em nenhum momento os alarmes sonoros do oxímetro foram desligados ou colocados em standby, exceto se o profissional de saúde já estivesse intervindo junto ao bebê.

Para a coleta de dados foram elaborados dois instrumentos. O primeiro destinado a coletar dados pertinentes à identificação, características e particularidades do bebê estudado. O segundo gerado automaticamente pelo software do sistema, que registrava os dados de identificação do bebê, seguido do registro dos valores da FP e SpO2 a cada 2 segundos, enquanto o bebê estivesse em observação. Os arquivos gerados foram transcritos para o software Excel, da Microsoft Corporation, organizados e analisados por estatística descritiva.

O projeto foi aprovado pelo Comitê de Ética em Pesquisa da Faculdade Evangélica do Paraná sob o protocolo No 3049/05. Obteve-se a autorização, por escrito, das Instituições e do coordenador de cada UTIN. Quando selecionado, o RN era realizado o contato com os pais, para esclarecimentos sobre o experimento atendendo à exigência da Resolução $\mathrm{N}^{\circ}$ 196/96 do Conselho Nacional de Saúde, e assinatura do Termo de Consentimento Livre e Esclarecido. Cada bebê integrante da pesquisa recebeu um número de controle em sua ficha de inscrição na pesquisa, sendo preservada sua identidade.

Salienta-se que o bebê, ao ser submetido ao experimento, era monitorado de acordo com o protocolo da unidade e continuava a receber o atendimento, conforme o estabelecido em cada UTIN, sem influência do experimento, nem interferência da pesquisadora no processo assistencial.

\section{RESULTADOS E DISCUSSÃO}

\section{Concepção e desenvolvimento do sistema}

O sistema de detecção e reversão do episódio apnéico denominado Anjo (Figura 1), é composto por um conjunto de equipamentos - Notebook marca Acer, modelo Travelmate $210 \mathrm{TXR}$ com processador Celeron 700; Oxímetro da marca Nellcor modelo 595; Vibrador Modelo VBW32 da Skin Transducer da Audiological Engineering; software desenvolvido para gerenciar o sistema e circuito eletrônico de isolação óptica da rede elétrica. $\mathrm{O}$ sistema foi concebido e desenvolvido por pesquisadores do Laboratório de Engenharia da Reabilitação da Pontifícia Universidade Católica do Paraná.

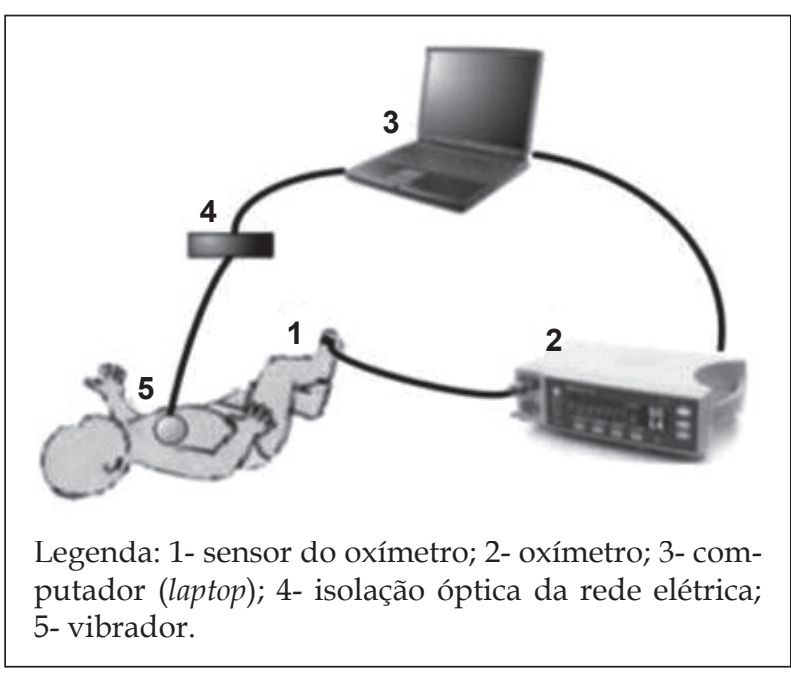

Figura 1 - Diagrama do setup de detecção e reversão do episódio de apnéia

O funcionamento do sistema prevê: a) o sensor do oxímetro que capta do bebê o sinal obtido por meio de transdução óptica de $\mathrm{FP}$ e $\mathrm{SpO}_{2}$; b) o oxímetro que processa esses sinais e os apresenta de forma numérica em um display; c) o oxímetro que envia para o computador as informações provindas do bebê pela interface serial RS 232; d) o computador que recebe os sinais de $\mathrm{FP}_{\mathrm{SpO}}$ na forma digital e os trata a partir de um programa que gerencia a ativação, ou não do vibrador, assim como registra os valores de $\mathrm{SpO}_{2}$ e FP durante o experimento; e) caso o bebê atinja os valores de $\mathrm{SpO} 2$ e FP estabelecidos para este estudo como marco de uma apnéia, o vibrador é acionado automaticamente, salientando-se, que a vibração só é gerada quando os dois parâmetros atingem os limites dos valores configurados.

Para o gerenciamento do sistema foi desenvolvido um software num ambiente de pro- 
gramação gráfica, o Laboratory Virtual Instrument Engineering Workbench (LabView) da National Instruments ${ }^{\circledR}$. O software contempla três módulos de atuação: módulo de vibração, módulo de estímulo manual, módulo de detecção da apnéia.

No módulo de vibração, o sistema ao detectar as condições de suporte a apnéia, aciona automaticamente o vibrador e registra os dados conforme sua configuração. Para este módulo foram desenvolvidas algumas funções para otimizar a forma de estimulação e de aquisição dos dados. Entre elas, a função que inibe a vibração e que pode ser reajustada, a qualquer momento, durante o experimento. Esta inibição é configurada para ocorrer no tempo estipulado em segundos e criada para evitar que o estímulo vibratório ocorra mais de uma vez, enquanto o bebê não atinja os limiares fisiológicos considerados normais.

Outra função desenvolvida relaciona-se ao estabelecimento do tempo de estímulo vibratório, para possibilitar ao pesquisador alterar o tempo de vibração em segundos, sempre em múltiplos de dois. Esta função expande as possibilidades de estudo do método de estímulo vibrotátil no futuro. Salienta-se que quando o módulo de vibração é ativado, o módulo de estímulo manual é automaticamente desligado e vice-versa.

O módulo de estímulo manual foi criado para gerar um registro do tempo de estimulação manual no RN realizado pelo profissional de saúde, que atua no cuidado ao bebê. É um módulocontrole e favorece a análise do comportamento do estímulo manual e estímulo vibrotátil durante o experimento. Para utilizar o módulo de estímulo manual é necessária a presença do observador, pois seu acionamento não é automático. Quando acionado, o sistema registra em intervalo de dois em dois segundos, que o bebê está recebendo estímulo manual, e prossegue até que o módulo seja desligado manualmente.

O módulo de detecção da apnéia foi desenvolvido para que seja registrado o tempo de apnéia do bebê, antes do início da estimulação manual. Esse módulo é acionado quando se aguarda a ocorrência da apnéia e não se pretende observar a estimulação manual. O módulo, quando acionado, inibe o funcionamento do módulo de estímulo vibratório. Foi criado para registrar a ocorrência e tempo de duração da apnéia. Uma particularidade de seu funcionamento é permitir a entrada do módulo de estímulo manual de forma concomitante, ou seja, ambos os registros acontecem, o do episódio de apnéia e o do tempo de duração da estimulação manual. O módulo de detecção da apnéia continua a registrar o episódio apnéico, até que os valores de $\mathrm{FPeSpO}$ alcancem os limites considerados normais para o RN. É importante mencionar que o registro do alarme da ocorrência da apnéia é constante, independentemente deste módulo estar ou não, acionado. A única diferença no comportamento do sistema ao se acionar o módulo de apnéia, é que o mesmo inibe o módulo de vibração, o que permite o registro do estímulo manual.

A interface do programa com o usuário desenvolvido para o sistema Anjo conta com três configurações: cabeçalho, configuração e gráficos. A primeira interface a ser preenchida é a do cabeçalho onde constam, o nome do arquivo para aquele bebê, o número do mesmo na pesquisa, a data de nascimento, idade gestacional e o grupo etário a que pertence. O sistema preenche automaticamente o diretório de gravação e a data da realização do experimento. Esses dados preenchidos pelo usuário constituem o cabeçalho de identificação do experimento no arquivo em formato texto, onde o mesmo grava a aquisição dos dados.

Na continuidade do experimento, a próxima tela a ser preenchida é a de configuração. Nesta tela, encontram-se os campos para ajustar os limiares de $\mathrm{FPe} \mathrm{SpO}_{2}$ que o sistema utiliza para acionar o vibrador, os registros de alarmes, o registro de apnéia, os limiares de gravação em estado não apnéico, o tempo de estímulo vibratório e o tempo de inibição do mesmo.

Ao se inicializar o software, os dados de configuração já se encontram preenchidos automaticamente na tela para um RN com idade gestacional menor ou igual a 35 semanas, podendo ser ajustada para estudar o bebê maior que 35 semanas. Essa tela tem as opções de preenchimento dos valores de $\mathrm{FP}$ e $\mathrm{SpO}_{2}$ que acionam o vibrador, o tempo de inibição da vibração e os valores mínimos de $\mathrm{FP} \mathrm{e} \mathrm{SpO}_{2}$ que o bebê apresentou durante o experimento.

O sistema não possui limitações de valores fixos, podendo ser utilizado no estudo do estímulo vibrotátil de outros indivíduos que não os RNs.

A interface gráficos, corresponde à tela que deve ser mantida aberta durante o experimento. Nela, é selecionado o módulo a ser utilizado naquele momento. É composta de alarmes visuais da ocorrência do registro do alarme de FP, de $\mathrm{SpO}_{2}$ ou de ambos, da ocorrência do estímulo vibrotátil e se o mesmo está sendo inibido. Apresenta, ainda, 2 gráficos de tendência que demonstram o comportamento dos parâmetros numa escala de 0 a 
$200 \mathrm{bpm}$ para a FP e de 0 a $100 \%$ para a $\mathrm{SpO}_{2}$. Ao lado dos gráficos, o valor numérico de $\mathrm{FP} \mathrm{eSpO}_{2}$ é visualizado. Esta tela ilustra os dados provenientes do oxímetro de pulso, tanto pelo visor do oxímetro quanto pela tela do software, o que permite a monitorização do bebê pela equipe da UTIN.

É importante salientar que o sistema registra apenas o estado de alarme da $\mathrm{FP}$ ou da $\mathrm{SpO}_{2}$, mas não é responsável pelo acionamento sonoro do mesmo, que são emitidos pelo oxímetro de pulso que, no início do experimento, é ajustado nos mesmos limiares do sistema. O registro destes pelo sistema independe do funcionamento dos alarmes do oxímetro e vice-versa.

Durante a coleta de dados um arquivo em formato texto é gerado automaticamente pelo sistema, registrando os dados provenientes do RN durante a realização do experimento.

No desenvolvimento do sistema adotou-se para a detecção da ocorrência do episódio apnéico, a determinação dos parâmetros fisiológicos e seus respectivos valores $\mathrm{FP}$ e $\mathrm{SpO}_{2}$. Para o estabelecimento dos valores diagnosticadores de ambos os parâmetros foram considerados os valores apontados na literatura, ${ }^{11}$ bem como os recomendados e adotados por três neonatologistas, coordenadores dos serviços envolvidos no estudo. Foram estabelecidos para $\mathrm{RN}<35$ semanas gestacionais a FP em 100 bpm e $\mathrm{SpO}_{2}$ em $80 \%$ e para $\geq 35$ semanas gestacionais em 80 bpm e $\mathrm{SpO}_{2}$ em $80 \%$.

\section{Experimento com recém-nascidos}

O experimento envolveu quatro RNs e 18 episódios apnéicos, sendo observados e aplicados o estímulo vibratório em 10 reversões e o estímulo manual em 8.

O método de reversão do episódio apnéico por meio do estímulo vibrotátil desencadeou movimentos respiratórios em nove das 10 apnéias nos quatro pré-termos observados, conforme Tabela 1.

Tabela 1 - Resultado da aplicação do estímulo vibratório nos recém-nascidos. Curitiba-PR, 2006

\begin{tabular}{|c|c|c|c|}
\hline Recém-nascido & Estímulo vibratório & $\begin{array}{l}\text { Retorno dos movi- } \\
\text { mentos respiratórios }\end{array}$ & $\begin{array}{c}\text { Retorno dos parâmetros de } \\
\text { freqüência de pulso e saturação } \\
\text { de oxigênio }\end{array}$ \\
\hline
\end{tabular}

\begin{tabular}{crrr}
\hline 1 & 2 & 2 & 2 \\
2 & 1 & 1 & 1 \\
3 & 2 & 2 & 1 \\
4 & 5 & 4 & 1 \\
\hline Total & 10 & 9 & 5 \\
\hline
\end{tabular}

Um trabalho realizado em Hatford, Connecticut, EUA, mostrou a utilização do estímulo vibrotátil para interrupção da apnéia no RN, com um RN em 18 apnéias. O estudo apresenta diferenças metodológicas em relação a presente pesquisa, relacionadas a: critério de inclusão dos sujeitos da pesquisa, tempo de estimulação vibratória, elenco de parâmetros diagnosticadores do episódio apnéico e os critérios considerados de sucesso para o retorno da respiração. Seis parâmetros fisiológicos foram considerados para a detecção do episódio apnéico: eletrocardiograma, frequência cardíaca, frequência de pulso, frequência respiratória, fluxo de ar nasal e curva pletismográfica de $\mathrm{SpO}_{2}$. $\mathrm{O}$ estímulo vibratório obteve sucesso em $56 \%$ das tentativas de reversão das apnéias. ${ }^{12}$
No presente estudo, foram utilizados somente dois parâmetros fisiológicos a $\mathrm{FP}$ e a $\mathrm{SpO} 2$, que se mostraram eficazes nesta detecção. A tecnologia proposta pode gerar vantagens, por utilizar, somente, o oxímetro de pulso para a detecção da apnéia, um equipamento rotineiramente incorporado no manejo da apnéia nas terapias intensivas em nosso meio, agregando um novo valor tecnológico, quando acoplado ao sistema de reversão da apnéia.

Cabe salientar que, embora os valores de FPe SpO2, configuradores da apnéia tenham sido adotados com base na literatura e na recomendação dos neonatologistas consultados, observou-se que os bebês, ao apresentarem esses valores, já manifestavam a ausência de movimentos respiratórios momentos antes. 
Tal achado gera a necessidade de investigações que possam indicar a necessidade de intervenção com o estímulo vibratório ou manual, mais precocemente. Para tanto, considerar a possibilidade de estabelecer outros valores de SpO2 e FP, superiores aos estipulados para o desencadeamento do estímulo, o que demandaria a utilização de um algoritmo que detecte e registre o momento da interrupção dos movimentos respiratórios. Com isso, seria possível verificar o intervalo entre a manifestação da apnéia e a variação da frequiência cardíaca e saturação de oxigênio, frente a esse epi- sódio. Avaliando essa relação de tempo de apnéia e resposta do organismo, podem ser estabelecidos limiares (ou variações) de FP e SpO2, que diagnostiquem a apnéia mais precocemente.

Quando da utilização da estimulação manual para a interrupção do episódio apnéico, nas oito apnéias, em que este estímulo foi aplicado, observou-se o retorno dos movimentos respiratórios em $100 \%$ delas. Em relação ao retorno dos parâmetros de FP eSpO2 após a aplicação do estímulo manual, sete das oito apnéias observadas conseguiram ser revertidas plenamente.

Tabela 2 - Resultado do experimento com o estímulo manual aplicados nos recém-nascidos. CuritibaPR, 2006

\begin{tabular}{cccc}
\hline Recém-nascido & Estímulo manual & $\begin{array}{c}\text { Retorno dos movi- } \\
\text { mentos respiratórios }\end{array}$ & $\begin{array}{c}\text { Retorno dos parâmetros de } \\
\text { frequência de pulso e saturação } \\
\text { de oxigênio }\end{array}$ \\
\hline 1 & 1 & 1 & 1 \\
2 & 1 & 1 & 1 \\
3 & 2 & 2 & 2 \\
4 & 4 & 4 & 3 \\
\hline Total & $\mathbf{8}$ & $\mathbf{8}$ & $\mathbf{7}$ \\
\hline
\end{tabular}

Observa-se que a estimulação manual conseguiu o retorno dos movimentos respiratórios em todos os episódios observados. Embora seja o procedimento tradicionalmente eleito para o manejo da apnéia, demanda mudanças que possam diminuir a manipulação requerida para tal estimulação.

Os estímulos dolorosos e táteis têm respostas hemodinamicamente específicas no córtex somatosensório e isto implica numa percepção sensória consciente do $\mathrm{RN}$ prematuro. ${ }^{13}$ A sensação tátil pode ser fonte de dor. ${ }^{14} \mathrm{O}$ estímulo manual pode causar desconforto, estresse e alterações cardiovasculares. ${ }^{15}$ Manipulações excessivas no RNPT podem causar estresse decorrente do frio, elevação da pressão arterial e aumento do fluxo sanguíneo cerebral com risco de hemorragia. ${ }^{16}$ Tais afirmações reforçam a importância da continuidade deste estudo para aprimoramento desta tecnologia.

\section{CONSIDERAÇÕES FINAIS}

A interrupção do episódio apnéico é um desafio enfrentado no cotidiano dos profissionais cuidadores do recém-nascido prematuro. Evidenciou-se no decorrer desse trabalho que há três requisitos básicos para o sucesso da reversão da apnéia: detecção precoce do episódio, imediata estimulação do recém-nascido e forma correta de estimulá-lo.

Os resultados apresentados não são conclusivos e demandam continuidade, com um conjunto amostral mais representativo para avaliar sua real eficiência. No entanto, algumas contribuições técnico-científicas podem ser extraídas deste estudo:

- que a interrupção do episódio apnéico, por meio do estímulo manual ocorre sem o estabelecimento de um protocolo;

- a possibilidade de desenvolvimento de protótipo para detecção do episódio apnéico baseado nos parâmetros e limiares de $\mathrm{FP}$ e SpO2;

- que o sistema de detecção de apnéia consegue detectar todos os episódios apnéicos;

- a possibilidade de desenvolvimento de protótipo para reversão do episódio apnéico baseado na utilização de estimulação vibrotátil;

- que o sistema de reversão do episódio apnéico mostra-se uma forma promissora do manejo da apnéia do recém nascido; 
- que os RNs reagiram a cada tipo de estímulo, vibratório e manual, de forma diferenciada, baseado na observação do comportamento do restabelecimento dos parâmetros fisiológicos de FP e SpO2, após a aplicação do estímulo;

- que o estímulo vibrotátil mostrou-se pior que o estímulo manual, quando comparados os tempos para a recuperação da $\mathrm{SpO} 2$ e FP após o retorno dos movimentos respiratórios;

- que o estímulo manual apresenta um melhor resultado de reversão completa da apnéia se comparado ao estímulo vibratório;

- que o estímulo vibratório mostra-se "mais confortável" na reversão da apnéia, e, consequentemente, mais benéfico ao recém-nascido quando consideradas as possíveis complicações geradas pela estimulação sensória do recém-nascido.

A necessidade de novas tecnologias para o cuidado do RN com apnéia, requer o desenvolvimento de tecnologias com custo exeqüível para o atendimento do RN brasileiro, e que contenham requisitos de segurança aliados à humanização, contemplando a minimização de possíveis complicações decorrentes do manejo da apnéia da prematuridade.

O método de reversão do episódio apnéico por meio do estímulo vibrotátil, atuando na frequência de $250 \mathrm{~Hz}$, durante quatro segundos mostrou um desempenho que se apresenta como uma forma promissora de manejo da apnéia da prematuridade.

Apesar de o trabalho ter envolvido uma pequena amostra de bebês, vislumbra, já nessa fase, promessa de se tornar um método inovador no manejo da apnéia no pré-termo.

Os resultados obtidos até o momento, apontam o caminho para a continuidade desse estudo. Acredita-se que este trabalho possa ser expandido para o manejo das várias faces da apnéia infantil, como aquelas decorrentes de infecção, anemia, síndrome da morte súbita do lactante, etc. Enfim, os achados abrem um leque de opções para pesquisas que busquem o melhor cuidado ao RNPT apnéico.

A tecnologia desenvolvida nessa pesquisa vem servir de instrumento para a realização do cuidado com qualidade, uma expressão material do pensamento crítico sobre a forma de prestação do cuidado. É uma forma de manifestação da humanização no atendimento, posto ter sido gerada sob a ótica de uma resposta crítica e responsável a uma necessidade encontrada no cuidado ao RN.
É imperiosa a necessidade do envolvimento do profissional enfermeiro em estudos de desenvolvimento de produtos destinados à melhoria do cuidado. Almeja-se que este profissional possa, além de prestar o cuidado direto, contribuir cientificamente para o desenvolvimento de novas tecnologias para aprimoramento do cuidado, àqueles que chegam a este mundo sem respeitar sua agenda fisiológica e estréiam na vida sem estar prontos, os pré-termos.

\section{REFERÊNCIAS}

1. Ministério da Saúde (BR), Secretaria de Políticas de Saúde, Área de Saúde da Criança. Atenção humanizada ao recém-nascido de baixo-peso: método mãe-canguru: manual do curso. Brasília (DF): MS; 2002.

2. Cloherty JP, Stark AR. Manual de neonatologia. $4^{\mathrm{a}}$ ed. Belo Horizonte (MG): Medsi; 2000. p. 42-3.

3. Sarmento, GJV. Peixe, AAF. Carvalho, FA. Fisioterapia respiratória em pediatria e neonatologia. Barueri (SP): Manole. 2007. p. 296.

4. Martin RJ, Abu-Shaweesh JM. Control of breathing and neonatal apnea. Biol Neonate. 2005 Jun; 87(4):288-95.

5. Boyce JM, Didier P. Guideline for hand hygiene in health-care settings. Recommendations of the Healthcare Infection Control Practices Advisory Committee and the HICPAC/SHEA/APIC/IDSA Hand Hygiene Task Force. Infect Control Hosp Epidemiol. 2002 Dec; 23(12 Suppl):S3-40.

6. Hellerud BC, Storm H. Skin conductance and behaviour during sensory stimulation of preterm and term infants. Early Hum Dev. 2002 Dec; 70(1-2):35-46.

7. Souza MWCR, Silva WCR, Araújo SAN. Quantificação das manipulações em recém-nascidos pré-termo em Unidade de Terapia Intensiva: uma proposta de elaboração de protocolo. Conscientiae Saúde. 2008; 7(2):269-74.

8. Lopes JMA. Neonatal apnea. J Pediatr. 2001; 77(s1):97-103.

9. Sreenan C, Lemke RP, Hudson-Mason A, Osiovich H. High-Flow Nasal Cannulae in the Management of Apnea of Prematurity: a comparison with conventional nasal continuous positive airway pressure. Pediatrics. 2001 May; 107(5):1081-3.

10. Koerich MS, Backes DS, Scortegagna HM, Wall ML, Veronese AM, Zeferino MT, et al. Tecnologias de cuidado em saúde e enfermagem e suas perspectivas filosóficas. Texto Contexto Enferm. 2006; 15(Esp):178-85.

11. Di Fiore JM, Arko MK, Miller MJ, Krauss A, Betkerur A, Zadell A, et al. Cardiorespiratory events in preterm infants referred for apnea monitoring studies. Pediatrics. 2001 Dec; 108(6):1304-8. 
12. Pichardo RL, Adam JS, Rosow E, Bronzino J, Eisenfeld L. Vibrotactile stimulation System to treat Apnea of prematurity. Biomed Instrum Technol. 2003 Jan-Feb: 37(1):34-40.

13. Bartocci M, Bergqvist LL, Lagercrantz H, Anand KJ. Pain activates cortical areas in the preterm newborn brain. Pain. 2006 May 122(1-2):109-17.

14. Hellerud BC, Storm H. Skin conductance and behaviour during sensory stimulation of preterm and term infants. Early Hum Dev. 2002 Dec; 70(1-2):35-46.
15. Duara S. Entrevista concedida a Sociedade Brasileira de Perinatologia. In: Anais do $17^{\circ}$ Congresso Brasileiro de Perinatologia, 2001 Nov 10-14; Florianópolis, Brasil. Florianópolis (SC): Sociedade Brasileira de Pediatria; 2001.

16. Bada HS. Toque mínimo. In: Anais do $17^{\circ}$ Congresso Brasileiro de Perinatologia, 2001 Nov 10-14; Florianópolis, Brasil. Florianópolis (SC): Sociedade Brasileira de Pediatria; 2001. p.274. 